\title{
Bessere Steueranreize für Banken
}

\section{Laurenz Grabher}

\section{Relevanz}

Eigenkapital ist teuer. Daher haben die Banken einen Anreiz, beim Eigenkapital zu sparen, und finanzieren sich lieber mit Spareinlagen und anderem Fremdkapital. Mit geringeren Finanzierungskosten ist es leichter, den Kunden im Wettbewerb bessere Konditionen anbieten zu können. Die Besteuerung fördert die Verschuldung der Banken zusätzlich. Mit dem Zinsabzug wird das Fremdkapital steuerlich entlastet, das risikotragende Eigenkapital jedoch nicht. Das fördert die Verschuldung der Banken und Unternehmen und trägt zur Krisenanfälligkeit bei. Die Bankenregulierung will mit höheren Kapitalstandards die Eigenkapitalausstattung und damit die Krisenrobustheit des Bankensektors stärken. Da macht es wenig Sinn, wenn der Staat mit dem steuerlichen Schuldenanreiz das genaue Gegenteil tut.

\section{Quelle}

Martin-Flores, Jose und Christophe Moussu (2018), Is Bank Capital Sensitive to a Tax Allowance on Marginal Equity? Erscheint in: European Financial Management, doi:10.1111/eufm.12163.

Hohe Überschuldung und wenig Eigenkapital gelten nicht erst seit der Finanzkrise 2008 als einer der grössten Risikofaktoren im Bankensektor. Strengere Kapitalvorschriften wie etwa die Reform der Bankenregulierung „Basel III“ zielen darauf

L. Grabher $(\bowtie)$

Universität St.Gallen, St.Gallen, Schweiz

E-Mail: laurenz.grabher@student.unisg.ch

(C) Der/die Autor(en) 2021

C. Keuschnigg und M. Kogler (Hrsg.), Die Wirtschaft im Wandel, https://doi.org/10.1007/978-3-658-31735-5_8 
ab, die Eigenkapitalquote der Banken zu erhöhen und so ihre Krisenrobustheit zu verbessern. Besonders strenge Kapitalvorschriften können aber auch zu einem Problem werden, wenn Banken ihre Kreditvergabe verringern, um auf diesem Weg die Eigenkapitalquote zu erhöhen. Das würde zu einer Kreditklemme führen und könnte ganz besonders in Krisenzeiten eine Rezession verschärfen.

Die Politik kann jedoch auch einen zweiten Weg gehen, die Krisenrobustheit der Banken zu stärken, ohne das Risiko einer Kreditklemme heraufzubeschwören. Sie könnte steuerliche Anreize setzen, damit Banken mehr Eigenkapital aufnehmen, anstatt sich hauptsächlich über Einlagen und anderes Fremdkapital zu refinanzieren. Wie andere Unternehmen können auch die Banken die Zinszahlungen auf ihr Fremdkapital von der Unternehmenssteuer abziehen. Gleichzeitig ist dies für die Eigenkapitalkosten nicht möglich. Dieser Steuernachteil schafft einen Anreiz, dass Banken ihre Eigenkapitalquote niedrig halten und sich lieber mit Fremdkapital finanzieren. Dies widerspricht dem Ziel der Bankenregulierung und fördert die Überschuldung. Ein gutes Anreizsystem dreht den Spiess um. Eigenkapital wird steuerlich nicht mehr länger benachteiligt, damit es eine attraktivere Finanzierungsquelle wird. So könnte eine Steuerreform die Banken unterstützen, mehr Eigenkapital zu bilden, damit sie Kreditausfälle und Verluste besser verkraften, Abschwünge abfedern und eine Krise leichter überstehen können.

Jose Martin-Flores und Christophe Moussu untersuchen, wie sich eine Steuerreform, welche die Diskriminierung des Eigenkapitals verringert, auf Eigenkapital und Verschuldung der Banken auswirkt. Dazu betrachten sie zwei Steuerreformen in Italien Ende der 1990er bzw. Anfang der 2000er Jahre. Sie zeigen, dass ein Abbau steuerlicher Nachteile die Eigenkapitalquote der Banken erhöht.

Italien führte mit der Steuerreform von 1998 einen Steuerabzug für Eigenkapital ein: Unternehmen und Banken zahlen eine reduzierte Gewinnsteuer auf das zusätzliche Eigenkapital, welches nach 1996 aufgenommen wurde, von $19 \%$ anstelle der vorher üblichen $37 \%$. Eigenkapital wurde steuerlich attraktiver. Wie wirkte sich diese Reform auf die Eigenkapitalbildung italienischer Banken aus? Um diesen Effekt zu schätzen, nutzen die Forscher die Tatsache, dass die anderen Mitglieder der Eurozone Ende der 1990er Jahre keine solche Steuerreform durchführten.

Konkret suchen sie zu den italienischen Banken eine Kontrollgruppe ganz ähnlicher Banken in den anderen Ländern. Was ähnlich ist, sollte auch ganz ähnlich auf wirtschaftliche Anreize reagieren. Wenn die Möglichkeit des Zinsabzugs auf Eigenkapital der einzige Unterschied zwischen den beiden Gruppen wäre, könnte man aus einer stärkeren Zunahme des Eigenkapitals italienischer Banken im Vergleich zur Kontrollgruppe der Banken anderswo auf einen positiven Effekt des Steueranreizes schliessen. Allerdings gibt es auch weitere Einflussgrössen auf die Kapitalstruktur von Banken wie Zinsen, Inflation oder Wirtschaftswachstum, die sich ebenfalls zwischen den Ländern unterscheiden. Indem sie auch diese Ein- 

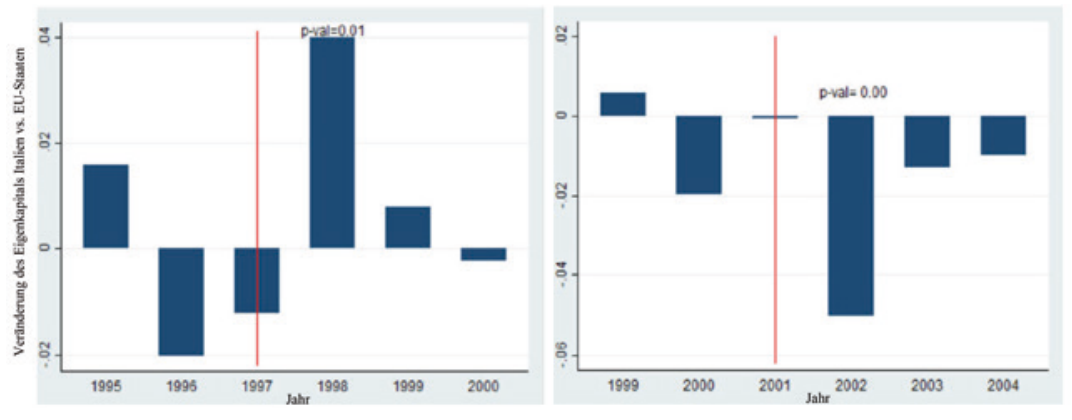

Abb. 1 Veränderung der Eigenkapitalquoten italienischer Banken relativ zu anderen Banken der Eurozone. (Quelle: Martin-Flores und Moussu 2018)

flüsse berücksichtigen und herausfiltern, können sie den Effekt des Steueranreizes in Italien isolieren. Als zentrales Ergebnis halten sie fest:

Die Einführung der Steuererleichterung erhöhte die Eigenkapitalquote italienischer Banken um 8.8 Prozent. Im Durchschnitt hatten somit italienische Banken im Vergleich zur Kontrollgruppe eine um 0.5 Prozentpunkte höhere Eigenkapitalquote einzig aufgrund der Steuerreform.

Die durchschnittliche Eigenkapitalquote italienischer Banken erhöhte sich um 8,8 \% im Vergleich zur Entwicklung ähnlicher Banken in anderen Ländern, die keine Steuerentlastung auf Eigenkapital einführten. Somit stieg die Eigenkapitalquote von 8,90 auf 9,43\%. Der linke Teil in Abb. 1 zeigt die Auswirkung sehr gut. Im Jahr nach der Einführung der Steuerentlastung im Jahr 1997 (vertikale, rote Linie) folgte ein erheblicher Anstieg der Eigenkapitalquote im Vergleich zu ähnlichen Banken in anderen Ländern. Die Forscher stellen fest, dass diese Veränderung darauf zurückgeht, dass die Banken zusätzliches Eigenkapital aufnahmen und nicht ihr Fremdkapital oder gar die Kreditvergabe verringerten.

Allerdings schaffte Italien die Steuerentlastung des Eigenkapitals mit Wirkung ab dem Jahr 2002 wieder ab. Martin-Flores und Moussu finden, dass der Effekt nun genau umgekehrt war, wie der rechte Teil der Abb. 1 zeigt. Nach dem Wiederaufleben der steuerlichen Benachteiligung des Eigenkapitals fielen im Jahr 2002 die Eigenkapitalquoten wieder um durchschnittlich 4,6\%, relativ zu den europäischen Vergleichsbanken. Die Banken passen also ihre Kapitalstruktur laufend an, wenn sich die (steuerlichen) Kosten des Fremd- und Eigenkapitals ändern. Ein Anstieg Eigenkapitalquote nach einem vorübergehenden Steueranreiz ist nicht langlebig, sondern verschwindet, wenn die Steuerbegünstigung wieder abgeschafft wird. 
Nach Abschaffung der Steuerbegünstigung des Eigenkapitals im Jahr 2001 sank in Italien die durchschnittliche Eigenkapitalquote ab 2002 um 4.6 Prozent relativ zu den europäischen Vergleichsbanken.

Kleine Banken mit einer Bilanzsumme unter 1 Mrd. EUR reagieren besonders stark auf die steuerlichen Anreize. Die Forscher identifizieren zwei mögliche Gründe dafür. Die erste Erklärung ist, dass grössere Banken international tätig sind und über zahlreiche Möglichkeiten verfügen, die Steuerbelastung in der Bankengruppe zu minimieren, z. B. durch Gewinnverschiebung in steuergünstige Länder. Eine weitere Steuerentlastung auf Eigenkapital wirkt sich dann nicht mehr so stark aus. Da kleinere Institute solche Möglichkeiten nicht haben, reagieren sie tendenziell stärker auf nationale Steueränderungen. Ihr zweiter Erklärungsversuch geht dahin, dass multinationale Banken als «too big to fail» bzw. systemrelevant gelten. Solche Banken profitieren von einer implizierten Staatsgarantie und reagieren weniger stark auf Unterschiede in nationalen Steuersystemen. Vielmehr spielen Faktoren wie z. B. die Kosten einer möglichen Insolvenz oder Eigenkapitalvorschriften eine Rolle.

Die steuerliche Diskriminierung des Eigenkapitals ist einer der Haupttreiber für die niedrige Kapitalausstattung und hohe Verschuldung von Banken und trägt so zur Krisenanfälligkeit von Banken bei. Reformen, welche den steuerlichen Nachteil des Eigenkapitals abbauen, können einen positiven Anreiz setzen und die Banken darin unterstützen, ihre Eigenkapitalquote deutlich zu erhöhen, wie das Beispiel Italiens Ende der 1990er Jahre zeigt. Wenn die Banken mehr Eigenkapital bilden, können sie auch mehr Kredite vergeben, ohne sich dabei selber mehr verschulden zu müssen. So kann die Steuerpolitik einen wachstumsfreundlichen Beitrag leisten, dass Banken ihre Eigenkapitalpuffer aufbauen und damit das Risiko von Bankinsolvenzen und Kreditklemmen in einem wirtschaftlichen Abschwung verringern.

Open Access Dieses Kapitel wird unter der Creative Commons Namensnennung 4.0 International Lizenz (http://creativecommons.org/licenses/by/4.0/deed.de) veröffentlicht, welche die Nutzung, Vervielfältigung, Bearbeitung, Verbreitung und Wiedergabe in jeglichem Medium und Format erlaubt, sofern Sie den/die ursprünglichen Autor(en) und die Quelle ordnungsgemäß nennen, einen Link zur Creative Commons Lizenz beifügen und angeben, ob Änderungen vorgenommen wurden.

Die in diesem Kapitel enthaltenen Bilder und sonstiges Drittmaterial unterliegen ebenfalls der genannten Creative Commons Lizenz, sofern sich aus der Abbildungslegende nichts anderes ergibt. Sofern das betreffende Material nicht unter der genannten Creative Commons Lizenz steht und die betreffende Handlung nicht nach gesetzlichen Vorschriften erlaubt ist, ist für die oben aufgeführten Weiterverwendungen des Materials die Einwilligung des jeweiligen Rechteinhabers einzuholen.

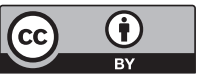

